\title{
Gathering Novel Circulating Exosomal microRNA in Osteosarcoma Cell Lines and Possible Implications for the Disease
}

\author{
Nicola Cuscino ${ }^{1,+}{ }^{\text {, Lavinia Raimondi }}{ }^{2,+}+\mathbb{D}$, Angela De Luca ${ }^{2}$, Claudia Carcione ${ }^{3}$, \\ Giovanna Russelli ${ }^{1}$, Laura Conti ${ }^{4}$, Jacopo Baldi ${ }^{5}$, Pier Giulio Conaldi ${ }^{1}$, \\ Gianluca Giavaresi ${ }^{2}$ (D) and Alessia Gallo ${ }^{1, *}$ \\ 1 IRCCS ISMETT (Istituto Mediterraneo per i Trapianti e Terapie ad alta specializzazione), \\ Research Department, 90127 Palermo, Italy; ncuscino@ismett.edu (N.C.); grusselli@ismett.edu (G.R.); \\ pgconaldi@ismett.edu (P.G.C.) \\ 2 IRCCS ISTITUTO ORTOPEDICO RIZZOLI, 40136 Bologna, Italy; lavinia.raimondi@ior.it (L.R.); \\ angela.deluca@ior.it (A.D.L.); gianluca.giavaresi@ior.it (G.G.) \\ 3 Fondazione Ri.MED, 90133 Palermo, Italy; ccarcione@fondazionerimed.com \\ 4 Body Fluids Biobank, Clinical Pathology, IRCCS Regina Elena Cancer National Institute, 00100 Rome, Italy; \\ laura.conti@ifo.gov.it \\ 5 Dept. of Orthopaedic Oncology, IRCCS Regina Elena National Cancer Institute, 00100 Rome, Italy; \\ jacopo.baldi@ifo.gov.it \\ * Correspondence: agallo@ismett.edu; Tel.: +39-9121-92649 \\ + The authors contributed equally to the study.
}

Received: 16 October 2019; Accepted: 28 November 2019; Published: 3 December 2019

\begin{abstract}
One of the goals of personalized medicine is to understand and treat diseases with greater precision through the molecular profile of the patient. This profiling is becoming a powerful tool for the discovery of novel biomarkers that can guide physicians in assessing, in advance, the disease stage, and monitoring disease progression. Circulating miRNAs and exosomal miRNAs, a group of small non-coding RNAs, are considered the gold standard diagnostic biomarkers for human diseases. We have previously demonstrated that osteosarcoma-derived exosomes are able to influence crucial mechanisms inside tumor niches, inducing osteoclast differentiation, and sustaining bone resorption activity. Here we discovered, through Next-Generation Sequencing (NGS), eight novel microRNAs in three different osteosarcoma cell lines, and assessed the selective packaging into the exosomes released. We then investigated, as proof-of-principle, the presence of the novel microRNAs in osteosarcoma patient samples, and found that 5 of the 8 novel microRNAs were more present in circulating exosomes of osteosarcoma patients compared with the controls. These results raise a question: Could the 8 novel microRNAs play a role for osteosarcoma pathogenesis? Although still premature, the results are encouraging, and further studies with a validation in a larger cohort are needed.
\end{abstract}

Keywords: microRNAs; exosomes; liquid biopsy

\section{Introduction}

Next-generation sequencing (NGS) and liquid biopsies, also through circulating microRNAs (miRNAs), are both tools that may provide therapeutic strategies to oncologists, which contribute to the development of precision medicine. Several research groups have already investigated circulating miRNAs for their diagnostic and prognostic potential, suggesting them as useful cancer biomarkers [1-4]. In osteosarcoma (OS), some clinical studies have investigated the prognostic and diagnostic potential 
of microRNAs [5,6]. Moreover, the identification of novel circulating miRNAs, released through exosomes into the blood from malignant cells, can provide novel biomarkers and therapeutic targets for cancer patients [6]. OS is the most common type of primary bone tumor of the skeleton, occurring mainly in children and adolescents, in the metaphyseal region of the long bones, typically in the distal femur, proximal tibia, and humerus [7].

Multidisciplinary treatment, combined with surgery for localized tumors, have led to a five-year survival rate of $60-70 \%$ in non-metastatic patients. However, approximately $20 \%$ of patients diagnosed with metastatic OS at presentation, primarily in the lungs, are instead characterized by a worse clinical outcome [8]. Among the novel approaches that do not require surgical biopsy, liquid biopsy is useful in improving the prognosis, and monitoring disease course and survival rates of OS patients, offering information on micro-metastasis at diagnosis and minimal residual disease, the latter only partially detectable by conventional diagnostic methods [9].

In our previous study, we highlighted, by RNA sequencing methods, a packaging of specific miRNAs within OS cell-derived exosomes. In detail, we first demonstrated the role of OS cell-derived exosomes, inside the tumor microenvironment, in bone metabolism and tumor angiogenesis; we then focused our attention on some of these specific exosomal miRNAs, and demonstrated their involvement in osteoclast differentiation, bone resorption activity, and angiogenesis [10].

In the present study, in taking advantage of the NGS approach, we discovered and analyzed the expression of eight novel miRNAs in OS cell lines and OS cell-derived exosomes. We also analyzed their expression in a panel of human tissues and in a small group of OS patients. Notably, we found selective packaging of some of these novel miRNAs into the exosomes released by OS cells and into the circulating exosomes from plasma of OS patients. Although, the functional role of exosome-encapsuled miRNAs must be investigated in depth, the results obtained lead us to hypothesize a role for these novel miRNAs as having a circulating biomarker potential in OS, and for enabling personalized treatments in precision medicine.

\section{Results}

\subsection{RNA Profiles of Osteosarcoma Cell Lines and Osteosarcoma Cell-Derived Exosomes}

In order to discover novel microRNA specific for OS, we start with three OS cell lines: SAOS-2, MG-63, U-2 OS, and the exosomes released by those cell lines. Exosomes were isolated and characterized as previously described [10]. After assessing the RNA quality, we performed small RNA sequencing on the MG-63 cell line and MG-63 exosomes; the U-2 OS cell line and U-2 OS exosomes; and the SAOS-2 cell line and SAOS-2 exosomes. Due to the NGS approach, we were able to determine, not only the differences in the expression of known miRNAs in the parent cells and the exosomes, but also novel sequences expressed among the different cell lines in relation to their exosome miRNA cargo. Novel miRNA profiles were analyzed in three OS cell lines: MG-63, U-2 OS, and SAOS-2, and their exosomes through miRDeep2. 3116, 5916, and 3381, novel putative miRNA sequences from MG-63, SAOS-2, and U-2 OS, respectively, remained after filtering for known contaminant and highly common sequences, such as rRNA and tRNA, for all known miRNA sequences from miRBase v22 and for exon (https://www.ncbi.nlm.nih.gov/sra/PRJNA575520). We further filtered the miRDeep2 results by score. MiRDeep2 scores ranged from -10 to 10, with a higher number corresponding to increased likelihood that the miRNA is genuine. A cut-off of 0 was used to be included in this study. We chose to validate two miRNAs for MG-63, three for U-2 OS, and three for SAOS-2, with the highest raw counts for each cell line (Table 1). In Supplementary Table S1 and Supplementary Material 2, we report the sequences, the genomic locations and predicted structures of the 8 pre-miRNA candidates. 
Table 1. Sequence and location information for the eight candidate miRNAs.

\begin{tabular}{cccc}
\hline Novel ID & Location (hg19) & Strand & Mature Sequence \\
\hline Candidate 1 & chr9:136204572..136204654 & - & CCCCACACUGCUAAAUUUGAC \\
Candidate 2 & chr3:14436198..14436257 & + & GGAAUAACGGGUGCUGUAGGCU \\
Candidate 3 & chr9:89037929..89037970 & - & CCCCUCACUGCUAAAUUUGAC \\
Candidate 4 & chrX:102411053..102411132 & - & CCAUCUGUGGGAUUAUGACUGA \\
Candidate 5 & chr16:33962833..33962878 & + & UGCGCAGUGGCAGUAUCGUAGCC \\
Candidate 6 & chr8:56821958..56822010 & - & UAUGUGCCUAGUGGCUGCUGUCU \\
Candidate 7 & chr13:27259470..27259536 & + & UCUGGGCAACAAGGUGAGACC \\
Candidate 8 & chr9:89037927..89037972 & - & AUGGAUUUUUGGAAAUAGGAGA \\
\hline
\end{tabular}

\subsection{Validation of the Candidates' Novel microRNAs}

Once we obtained the 8 candidate microRNA sequences (Table 1) with a high score through miRDeep2, we used custom Taqman assays to first validate on the SAOS-2, MG-63, and U-2 OS cell lines and their exosomes (Supplementary Figure S1). We then measured the expression of the candidate microRNAs across a panel of 10 different human tissue RNA (skeletal muscle, stomach, testis, kidney, lung, brain, prostate, liver, spleen, and bone) to assess the presence in different body parts and eventually the tissue specificity. Custom Taqman assays gave reproducible and consistent results, and were able to amplify the target novel miRNAs in most of the tissue types tested. The liver, kidney, and brain RNAs showed the highest candidate microRNAs expression, while skeletal muscle, lung, spleen and bone showed the lowest candidate microRNAs expression (Figure 1).
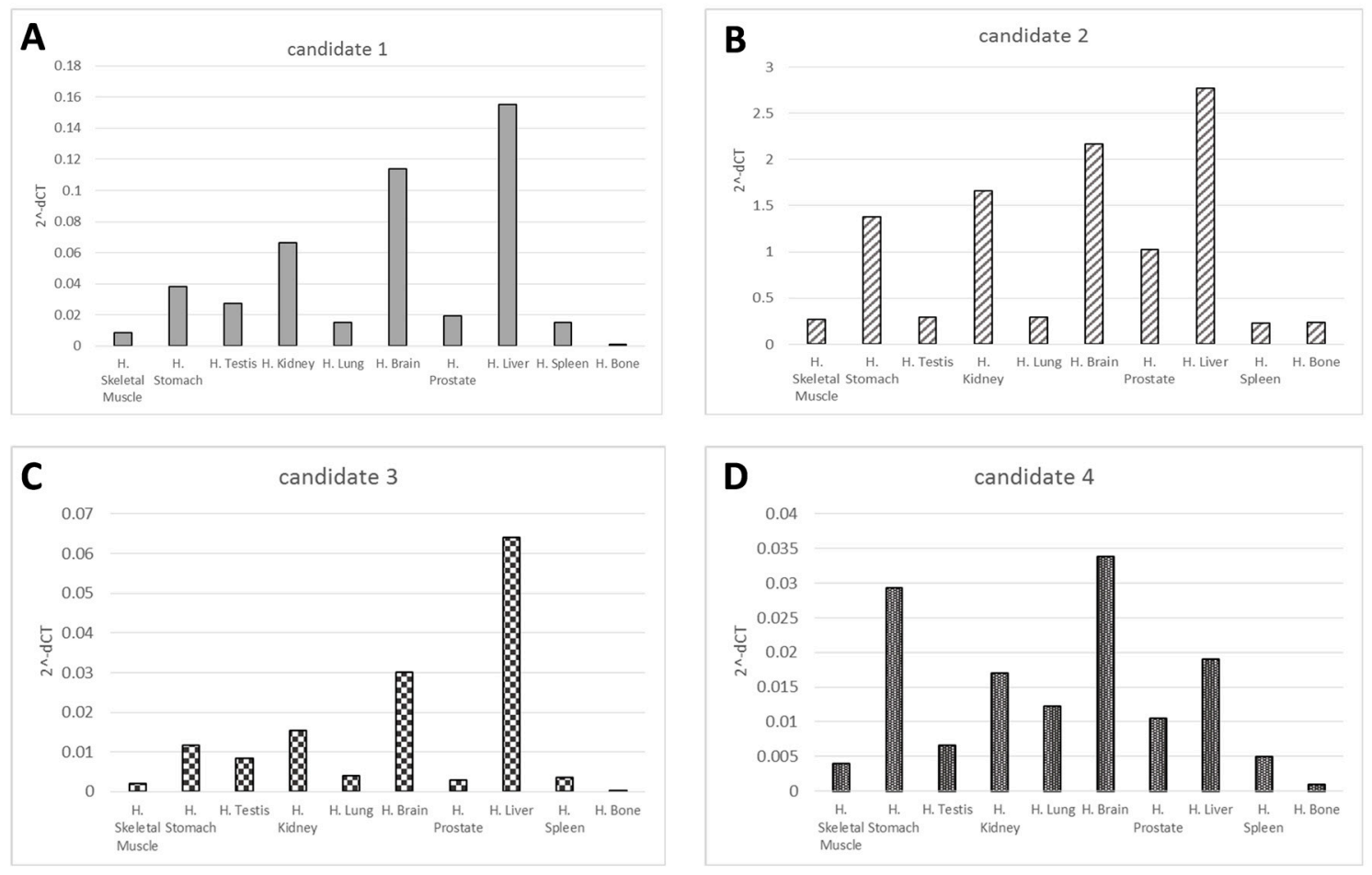

Figure 1. Cont. 

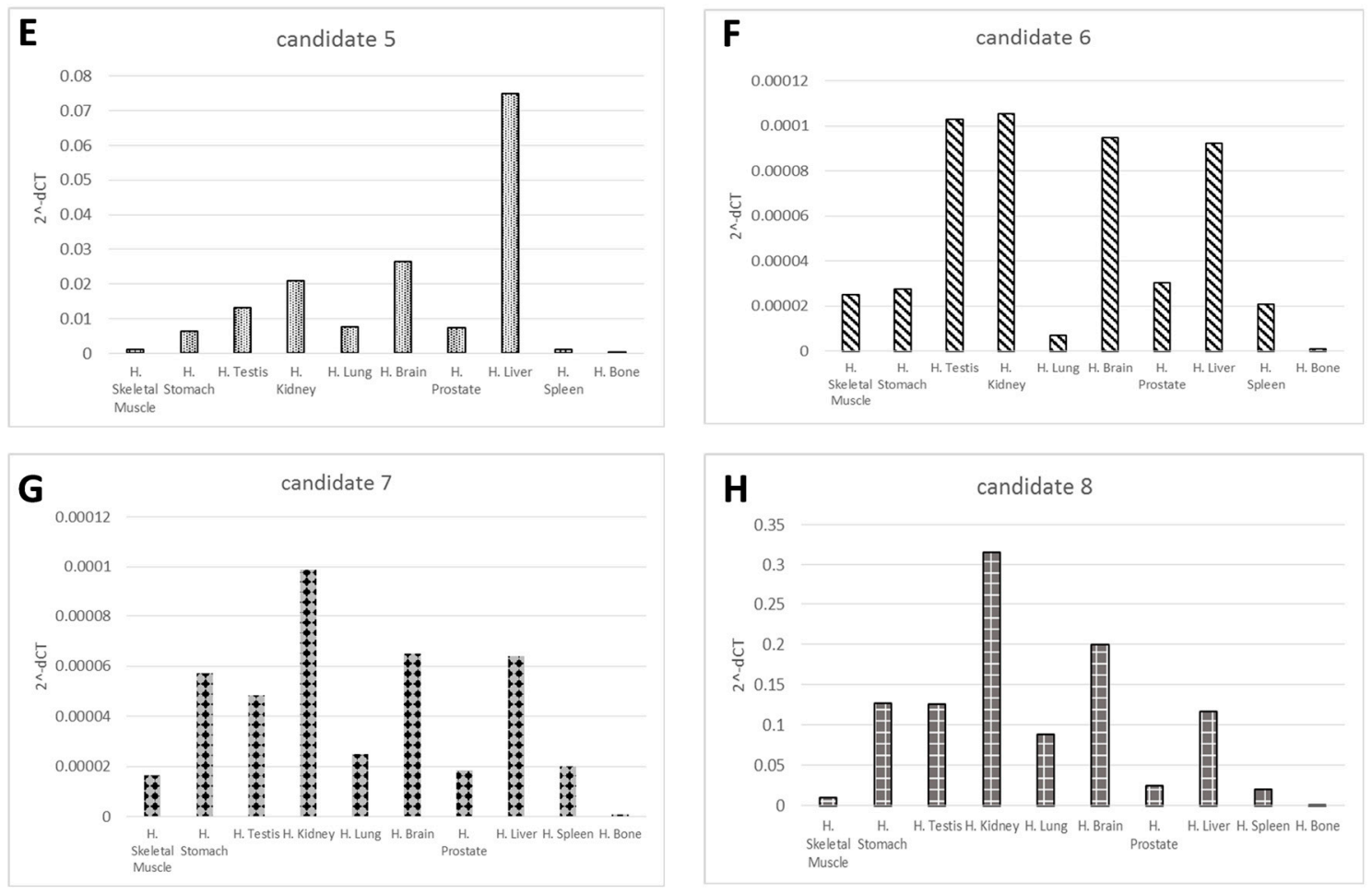

Figure 1. Novel candidate miRNA validation. The graphs (A-H) show quantitative RT-PCR results of the 8 novel candidate miRNAs expression, respectively, in 10 different human tissue types. Results are displayed as mean levels to the average expression reported as $2^{\wedge-\mathrm{dCT}}$, and are normalized to U6.

\subsection{Candidate Novel microRNA Expression Circulating in Osteosarcoma Samples}

Once validated in different human tissues, we wanted to investigate the presence of the candidate microRNAs discovered in OS cell lines, and eventually the differential in samples of OS patients. As proof-of-principle, to test the prognostic potential of the novel candidate microRNAs in liquid biopsies, we analyzed plasma from 5 OS patients whose clinical features are reported in Table 2, and 3 controls by digital PCR.

Table 2. Clinical characteristics of Osteosarcoma patients.

\begin{tabular}{|c|c|c|c|c|c|c|}
\hline Patient ID & Gender & Age & $\begin{array}{l}\text { Site of } \\
\text { Origin }\end{array}$ & $\begin{array}{l}\text { Histologic } \\
\text { Subtype }\end{array}$ & metastasis & Treatments \\
\hline Patient 1 & M & 14 & sx prox Tibia & $\begin{array}{l}\text { High Grade } \\
\text { Surface OS }\end{array}$ & No & Chir \\
\hline Patient 2 & M & 17 & $\begin{array}{l}\mathrm{dx} \text { distal } \\
\text { Femur }\end{array}$ & OS G3 & No & $\begin{array}{c}\text { CHT }(\text { CDDP/ADM } \\
\text { MTX HD } \times 2)\end{array}$ \\
\hline Patient 3 & M & 18 & $\mathrm{dx}$ prox Tibia & $\begin{array}{c}\text { OS condrobastic } \\
\text { G3 }\end{array}$ & No & $\begin{array}{c}\text { CHT }(\mathrm{CDDP} / \mathrm{ADM} \\
\text { MTX HD } \times 2)\end{array}$ \\
\hline Patient 4 & M & 19 & sx Tibia & OS fibroblastic & Lung & $\begin{array}{c}\text { CHT (CDDP/ADM } \\
\text { MTX HD } \times 2)\end{array}$ \\
\hline Patient 5 & M & 16 & $\mathrm{dx}$ Pelvis & OS G3 & Lung & $\begin{array}{c}\text { CHT }(\mathrm{CDDP} / \mathrm{ADM} \\
\text { MTX HD } \times 2)\end{array}$ \\
\hline
\end{tabular}

We choose this approach because of such advantages as the partitioning of the PCR reaction into thousands of individual reactions; the end-point measurement enables nucleic acid quantitation independent of the reaction efficiency. In this set of samples, 5 novel candidate microRNAs (candidates $2,4,5,6$, and 8) out of eight showed a significant differential expression in OS samples compared with the controls (Figure 2). One novel candidate microRNA (candidate 7) did not amplify in any sample; 
one novel candidate microRNA (candidate 3) showed no difference between the two groups, and novel candidate 1 showed the highest expression in the control group compared with the OS group.
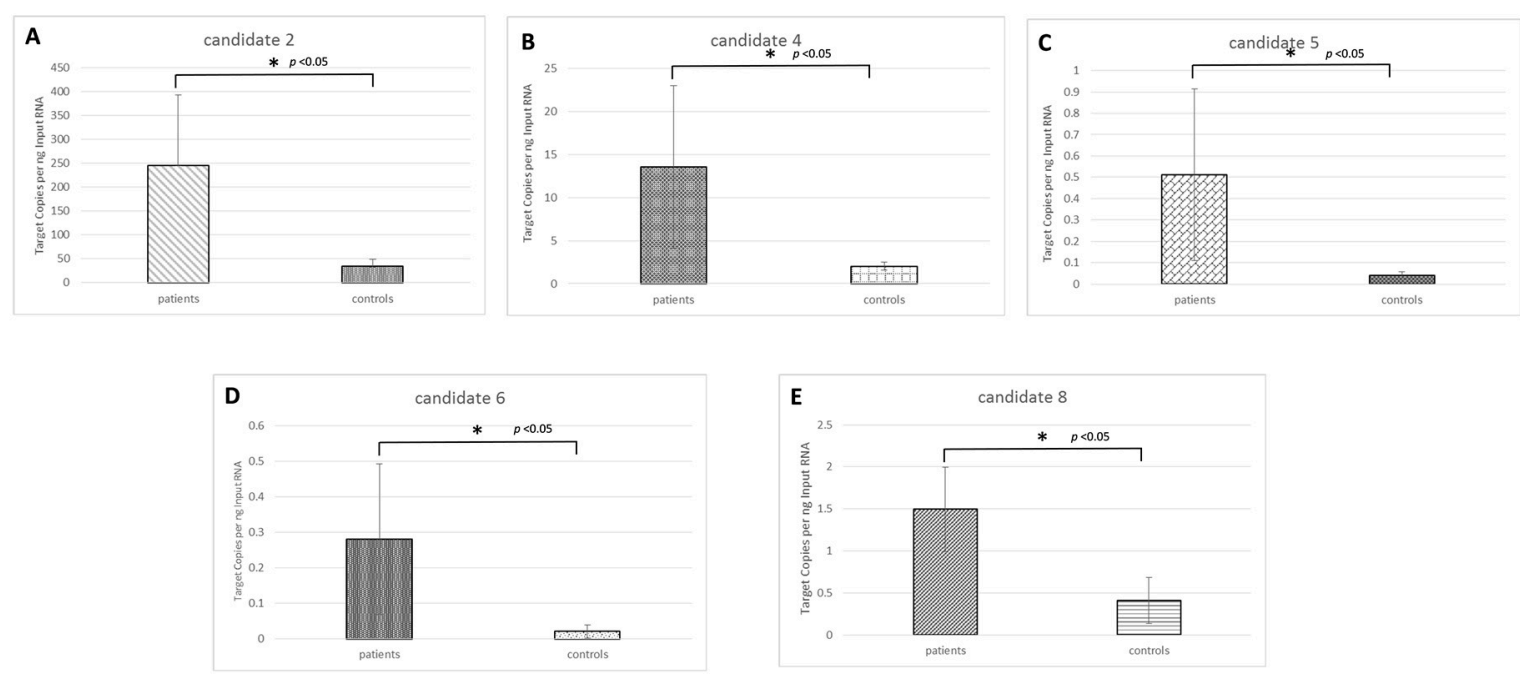

Figure 2. Novel candidate miRNAs expression in osteosarcoma patient samples. The graphs (A-E) show quantitative RT-PCR results of the novel candidate miRNAs 2/4/5/6/8 respectively that resulted highly expressed circulating in osteosarcoma sample compared with the controls. Results are displayed as mean levels to the average expression calculated as Target Copies per ng Imput RNA, and are normalized to U6. One star $\left(^{*}\right)$ indicates $p<0.05$, two-tailed Student's test.

We further determined the biologic pathways affected by novel candidate miRNAs using TargetScan web platform able to predict biological targets of microRNAs by searching for the presence of sites that match the seed region of each miRNA. We reported in Table 3 the KEGG biological processes significantly enriched (pathways with $p<0.05$ ). All clearly involved with carcinogenesis for each candidate microRNAs determined by Enrichr web platform. 
Table 3. Biologic pathways enriched by differentially expressed novel candidate miRNAs.

\begin{tabular}{|c|c|c|c|}
\hline Candidate 2 & KEGG pathway & $p$-value & Genes \\
\hline & FoxO signaling pathway & $6.37 \times 10^{-5}$ & SMAD2;SMAD4;CCND2;MAPK1;PIK3R1;IGF1 \\
\hline & TGF-beta signaling pathway & $1.208 \times 10^{-3}$ & SMAD2;SMAD4;MAPK1;ACVR1B;BMPR1B;SKP1 \\
\hline & Hippo signaling pathway & $1.29 \times 10^{-3}$ & FZD1;SMAD2;PRKCI;SMAD4;CCND2;NF2 \\
\hline & Proteoglycans in cancer & $1.41 \times 10^{-3}$ & FZD1;SMAD2;PDCD4;MAPK1;PIK3R1;IGF1 \\
\hline & Hepatocellular carcinoma & $1.75 \times 10^{-3}$ & FZD1;SMAD2;SMAD4;CDK6;MAPK1;PIK3R1 \\
\hline & Glioma & $3.09 \times 10^{-3}$ & CDK6;MAPK1;PIK3R1;IGF1;CALM1 \\
\hline & Pancreatic cancer & $3.09 \times 10^{-3}$ & SMAD2;SMAD4;CDK6;MAPK1;PIK3R1 \\
\hline & Breast cancer & $3.35 \times 10^{-3}$ & FZD1;JAG2;CDK6;MAPK1;PIK3R1;IGF1;ESR1 \\
\hline \multirow[t]{9}{*}{ Candidate 4} & KEGG pathway & $p$-value & Genes \\
\hline & Cell adhesion molecules & $3.61 \times 10^{-4}$ & NLGN3;CNTNAP1;CD4;NLGN2;PECAM1;NRXN3 \\
\hline & Focal adhesion & $6.73 \times 10^{-4}$ & PPP1CB;PPP1CC;RAP1A;COL4A4;ITGB8;MAPK1;CRK \\
\hline & TGF-beta signaling pathway & $1.08 \times 10^{-3}$ & SRC;MAPK1;CRK;CD44;PFN2 \\
\hline & Renal cell carcinoma & $1.12 \times 10^{-3}$ & PPP1CB;PPP1CC;FGF7;ITGB8;MAPK1;PIP4K2C;CRK \\
\hline & Rap1 signaling pathway & $1.32 \times 10^{-3}$ & PPP1CB;PPP1CC;PRKAB2;RAPGEF1;MAPK1 \\
\hline & ErbB signaling pathway & $4.50 \times 10^{-3}$ & ZFYVE16;SP1;MAPK1;INHBA;RGMB \\
\hline & VEGF signaling pathway & $8.46 \times 10^{-3}$ & PPP1CB;PPP1CC;RAP1A;MAPK1 \\
\hline & Proteoglycans in cancer & $9.37 \times 10^{-3}$ & RAP1A;RAPGEF1;MAPK1;CRK \\
\hline \multirow[t]{8}{*}{ Candidate 5} & KEGG pathway & $p$-value & Genes \\
\hline & Bladder cancer & $1.22 \times 10^{-2}$ & VEGFA \\
\hline & VEGF signaling pathway & $1.75 \times 10^{-2}$ & VEGFA \\
\hline & Renal cell carcinoma & $2.05 \times 10^{-2}$ & VEGFA \\
\hline & Pancreatic cancer & $2.22 \times 10^{-2}$ & VEGFA \\
\hline & HIF-1 signaling pathway & $2.69 \times 10^{-2}$ & VEGFA \\
\hline & Focal adhesion & $2.69 \times 10^{-2}$ & VEGFA \\
\hline & Proteoglycans in cancer & $2.96 \times 10^{-2}$ & VEGFA \\
\hline
\end{tabular}


Table 3. Cont.

\begin{tabular}{|c|c|c|c|}
\hline Candidate 6 & KEGG pathway & $p$-value & Genes \\
\hline & Proteoglycans in cancer & $2.45 \times 10^{-3}$ & PPP1R12A;ARHGEF12;FZD5;ERBB4;KDR;IGF1R \\
\hline & MAPK signaling pathway & $3.93 \times 10^{-3}$ & GABRB2;CACNA1C;GABARAP;TRAK2 \\
\hline & Focal adhesion & $1.13 \times 10^{-2}$ & PDGFRA;CACNB4;ERBB4;KDR;CACNA1C;IGF1R \\
\hline & Rap1 signaling pathway & $5.11 \times 10^{-2}$ & PDGFRA;PPP1R12A;KDR;PARVA;IGF1R \\
\hline \multirow[t]{8}{*}{ Candidate 8} & KEGG pathway & $p$-value & Genes \\
\hline & Hippo signaling pathway & $2,00 \times 10^{-4}$ & PРP1CB;LATS2;DLG3;FZD4;TCF7;YWHAZ;TGFBR1; \\
\hline & TGF-beta signaling pathway & $2.14 \times 10^{-3}$ & CREBBP;TCF7;BAIAP2;TGFBR1;WASF3 \\
\hline & Breast cancer & $5.62 \times 10^{-3}$ & CREBBP;RPS6KB1;NEO1;ACVR2B;TGFBR1 \\
\hline & Colorectal cancer & $7.87 \times 10^{-3}$ & HDAC4;BECN1;RPS6KB1;ADCY2;CALM2;TGFBR1 \\
\hline & Prostate cancer & $9.07 \times 10^{-3}$ & CREBBP;FZD4;TCF7;ADCY2;CALM2 \\
\hline & Gastric cancer & $1.09 \times 10^{-2}$ & RPS6KB1;FZD4;NCOA3;TCF7;PGR;LRP6 \\
\hline & Wnt signaling pathway & $2.19 \times 10^{-2}$ & NRP1;CREBBP;CDC27;ADCY2;TGFBR1;CREB5 \\
\hline
\end{tabular}




\section{Discussion}

In the management of OS, a proper diagnosis and staging of the disease is a major prerequisite for effective surgical and pharmacological treatments. Conventional diagnostic approaches, such as tissue biopsy and imaging, remain the most common diagnostic tests. Nevertheless, tissue biopsy may sometimes be difficult to obtain and not be easily repeatable. In addition, information concerning micro-metastasis or minimal residual disease can sometimes be lost with conventional diagnostic methods [11]. Liquid biopsy may be highly advantageous, given the difficulty of recovering the bone sample for disease staging. In fact, it is a non-invasive and also time-saving approach; moreover, it may offer more precise and accurate information on early detection, therapeutic decisions, and response to therapy in OS $[12,13]$.

In this paper we identified eight novel microRNAs in OS cell lines (MG-63, SAOS-2, and U-2 OS) and their released exosomes. The eight sequences remained after filtering for known contaminant from 3116, 5916, and 3381, novel putative miRNA sequences, obtained by MG-63, SAOS-2, and U-2 OS, respectively. We chose to validate two miRNAs for MG-63, three for U-2 OS, and three for SAOS-2, with the highest raw counts for each cell line. To assess the tissue specificity, we analyzed, by custom TaqMan assays, the expression of the eight candidate microRNAs across a panel of 10 different human tissue RNAs (skeletal muscle, stomach, testis, kidney, lung, brain, prostate, liver, spleen and bone). The candidate microRNAs were found highly expressed in the liver, kidney, and brain, while skeleton muscle, lung, spleen and bone showed the lowest candidate microRNAs expression.

To fill the current gaps and needs advanced by clinical oncologists, we decided to investigate the presence of the novel candidate microRNAs circulating in OS patients, and compare them with controls. Five of eight novel candidate microRNAs were found highly and significantly expressed in OS samples compared with the controls. This fact, together with the low expression of these microRNAs in the healthy bone tissue, make the novel candidates potential disease biomarkers.

Through TargetScan web platform, we identified the putative genes regulated by these five candidate microRNAs. We further determined, through the web platform Enrichr, the biologic pathways predicted and potentially affected. The KEGG biological processes significantly enriched (pathways with $p<0.05$ ), reported in Table 3 , resulted clearly involved with carcinogenesis for each candidate; Among these, candidate microRNA 5 is predicted to bind and strongly regulate VEGFA which expression is crucial for OS growth and metastasis [14]. Another example is candidate microRNA 2, predicted to bind SMAD2 and SMAD4, genes already related with Osteosarcoma [15], and resulting potentially interesting for further in vitro studies.

Overall, these results allowed us to hypothesize that these 8 novel candidate miRNAs could potentially represent biomarkers for OS. As a result, we plan to carry out future clinical studies on large cohorts of OS patients in order to better analyze and compare the expression of novel miRNAs in patient subgroups, distinguishing metastatic patients from non-metastatic ones. We suppose that such information could correlate the expression of miRNAs with the disease progression. At the same time, functional studies, by in vitro assays, may shed light on the molecular mechanisms beyond the possible pathologic role of these microRNAs.

\section{Materials and Methods}

\subsection{Ethics Approval}

The Bioethics Committee of the IFO_AOO - AOO - Istituti Fisioterapici Ospitalieri on February 16, 2017 (resolution number899/17) or osteosarcoma patients approved this study.

The aim of the study, study stages, and sample collection procedures were explained to all subjects. All subjects gave their written informed consent to participate in the study, including for blood sample collection and use of clinical data for research. 


\subsection{Cell Lines and Reagents}

SAOS-2, MG-63, and U-2 OS cell lines were purchased from ECACC (Sigma-Aldrich, Milano, Italy), and grown in DMEM high glucose (Thermo Fisher Scientific, Cambridge, MA, USA) supplemented with $10 \%$ fetal bovine serum (FBS, Lonza Group, Basel, Switzerland).

\subsection{Exosome Purification}

Exosomes released by OS cells (SAOS-2, MG-63, and U-2 OS) during a 48-hour culture period were isolated from conditioned culture medium supplemented with 10\% FBS, previously ultra-centrifuged by differential centrifugations as previously described. Exosome protein content was determined by the Bradford assay [16]. Exosomes from plasma of OS patients were isolated with Total Exosome Isolation Kit (Thermo Fisher Scientific, Cambridge, MA, USA), according to the manufacturer's instructions.

\subsection{Small RNA Library Construction and Sequencing}

To test the quality and assess the quantity of total RNA extracted, we used the RNA ScreenTape assay on a 2200 TapeStation system (Agilent Technologies, Santa Clara, CA, USA). For small RNA-Seq, $1 \mu \mathrm{g}$ of total RNA per sample was used for library preparation using TruSeq Small RNA Sample Prep Kits (Illumina, San Diego, CA, USA). Size-distribution was measured with the DNA ScreenTape assay on a 2200 TapeStation system (Agilent Technologies, Santa Clara, CA, USA). A total library pool of $4 \mathrm{nM}$ was sequenced using a MiSeq Reagent Kit v3 150 cycle on a MiSeq System (Illumina, San Diego, CA, USA).

\subsection{Small RNA-Seq in Silico Analysis}

We used the Trimmomatic software [17], v. 0.3633 to remove adaptors, low-quality bases, and reads with less than 16 nucleotides. The parameter "ILLUMINACLIP TruSeq3-SE.fa:2:30:10" was used to remove read adaptors according to Illumina-specific sequences. A sliding window cut was applied to remove bases with average quality below 22 using the parameter "SLIDINGWINDOW:3:22," and reads with less than 16 nucleotides were removed using "MINLEN:16."

Alignment of miRNA sequencing reads to the human reference genome build hg19 was performed using Bowtie v. 1.2.2 [18]. More stringent read length filtering was carried out by miRDeep2 [19] before the identification of novel miRNAs, discarding reads with length less than 18 nucleotides and greater than 23 nucleotides. We used the miRDeep2 v.2.0.1.1 software (Berlin Institute for Medical Systems Biology at Max-Delbrück-Center for Molecular Medicine, Berlin-Buch, Germany) default parameters, using the Fasta format sequences of all mature and hairpin miRNA sequences obtained from miRBASE v.22 database.

\subsection{Real-time PCR Validation and Digital PCR of Novel microRNAs}

For miRNA validation, total RNA from 10 human tissues (H. Skeletal Muscle, H. Stomach, H. Testis, H. Kidney, H. Lung, H. Brain, H. Prostate, H. Liver, H. spleen from Gentaur S.r.l. Italy and H. Bone from OriGene Technologies $\mathrm{GmbH}$, MD, USA) were reverse transcripted with TaqMan MicroRNA Reverse Transcription Kit, according to the manufacturer's instructions (Thermo Fisher Scientific, Cambridge, MA, USA). Custom TaqMan microRNA Assay are listed in Supplementary Table S2). Novel candidate miRNA expression results are displayed as $2^{\wedge-\mathrm{dCT}}$, and are normalized to U6 (Thermo Fisher Scientific, Cambridge, MA, USA). Twenty ng of RNA extracted from plasma of OS patients and controls were reverse transcripted with TaqMan MicroRNA Reverse Transcription, according to the manufacturer's instructions (Thermo Fisher Scientific, Cambridge, MA, USA). The Droplet Digital ${ }^{\mathrm{TM}}$ PCR (ddPCR) reactions were prepared in $20 \mu \mathrm{L}$ total volumes, according to the manufacturer's instructions (Bio-Rad Laboratories, Irvine, CA, USA). The thermal cycling conditions of the droplets generated were as follows: $95^{\circ} \mathrm{C}$ for $10 \mathrm{~min}(1 \mathrm{cycle})$; then 40 cycles of $94^{\circ} \mathrm{C}$ for $30 \mathrm{~s}$, and $60^{\circ} \mathrm{C}$ for $1 \mathrm{~min}, 98^{\circ} \mathrm{C}$ for $10 \mathrm{~min}$, and then held at $4{ }^{\circ} \mathrm{C}$. After thermal cycling, droplets were analyzed for positive and negative signals 
using the QX200 droplet reader (Bio-Rad Laboratories, Irvine, CA, USA). Data analysis was done when the number of droplets produced was more than 20,000. For data analysis, QuantaSoft Version 1.7.4 software (Bio-Rad Laboratories, Irvine, CA, USA) was used to statistically analyze the obtained data. Novel candidate miRNA expression was normalized on U6 (Thermo Fisher Scientific, Cambridge, MA, USA) and the results are displayed as Target Copies per ng Imput RNA [20].

\section{7. miRNA Pathway Analysis}

To identify potential target genes and pathways of the differentially expressed novel candidate miRNAs found, we conducted in silico analysis using TargetScan web platform [21], in order to identify the putative genes and Enrichr web platform [22] to determine the putative pathways.

\section{Conclusions}

We have identified eight novel microRNAs, through NGS, in three osteosarcoma cell lines and demonstrated the selective packaging into their released exosomes. Below, the expression of the novel miRNAs was confirmed in circulating exosomes from plasma of osteosarcoma patients. The exact role of the novel miRNAs will be assessed through future clinical and molecular studies. The data obtained suggest these novel miRNAs as having a circulating biomarker potential in osteosarcoma cancer, assuming a role in personalized medicine.

Supplementary Materials: The following are available online at http://www.mdpi.com/2072-6694/11/12/1924/s1, Figure S1: Novel candidate miRNA validation. Table S1: Sequence and location information for the eight pre-miR candidate. Table S2: List of Custom Assay ID for novel candidate miRNA validation. The sequencing raw data are available online at https://www.ncbi.nlm.nih.gov/sra/PRJNA575520.

Author Contributions: Conceptualization: N.C., L.R., and A.G.; methodology, G.R., A.D.L., L.C., and J.B.; software, N.C.; validation, C.C., G.R., and A.D.L.; formal analysis, N.C., L.R., and A.G.; investigation, A.G.; writing-original draft preparation, N.C., L.R., and A.G.; writing-review and editing, A.G., P.G.C., and G.G.; visualization, L.R. and A.G.; supervision, A.G., P.G.C., and G.G.

Funding: This research received no external funding.

Acknowledgments: We would like to thank Warren Blumberg of IRCCS ISMETT's Language Services Department for assistance in editing the manuscript.

Conflicts of Interest: The authors declare no conflict of interest. The funders had no role in the design of the study; in the collection, analyses, or interpretation of data; in the writing of the manuscript, or in the decision to publish the results.

\section{References}

1. Mitchell, P.S.; Parkin, R.K.; Kroh, E.M.; Fritz, B.R.; Wyman, S.K.; Pogosova-Agadjanyan, E.L.; Peterson, A.; Noteboom, J.; O’Briant, K.C.; Allen, A.; et al. Circulating Micrornas as Stable Blood-Based Markers for Cancer Detection. Proc. Natl. Acad. Sci. USA 2008, 105, 10513-10518. [CrossRef] [PubMed]

2. Chen, X.; Ba, Y.; Ma, L.; Cai, X.; Yin, Y.; Wang, K.; Guo, J.; Zhang, Y.; Chen, J.; Guo, X.; et al. Characterization of Micrornas in Serum: A Novel Class of Biomarkers for Diagnosis of Cancer and Other Diseases. Cell Res. 2008, 18, 997-1006. [CrossRef] [PubMed]

3. Hamam, R.; Hamam, D.; Alsaleh, K.A.; Kassem, M.; Zaher, W.; Alfayez, M.; Aldahmash, A.; Alajez, N.M. Circulating Micrornas in Breast Cancer: Novel Diagnostic and Prognostic Biomarkers. Cell Death Dis. 2017, 8, e3045. [CrossRef] [PubMed]

4. Sholl, L.M. Next-Generation Sequencing from Liquid Biopsies in Lung Cancer Patients: Advances in Comprehensive Biomarker Testing. J. Thorac. Oncol. 2017, 12, 1464-1466. [CrossRef] [PubMed]

5. Sarver, A.L.; Phalak, R.; Thayanithy, V.; Subramanian, S. S-Med: Sarcoma Microrna Expression Database. Lab. Investig. 2010, 90, 753-761. [CrossRef]

6. Brase, J.C.; Wuttig, D.; Kuner, R.; Sultmann, H. Serum Micrornas as Non-Invasive Biomarkers for Cancer. Mol. Cancer 2010, 9, 306. [CrossRef]

7. Ottaviani, G.; Jaffe, N. The Etiology of Osteosarcoma. Cancer Treat. Res. 2009, 152, 15-32. 
8. Marina, N.; Gebhardt, M.; Teot, L.; Gorlick, R. Biology and Therapeutic Advances for Pediatric Osteosarcoma. Oncologist 2004, 9, 422-441. [CrossRef]

9. Palmirotta, R.; Lovero, D.; Cafforio, P.; Felici, C.; Mannavola, F.; Pelle, E.; Quaresmini, D.; Tucci, M.; Silvestris, F. Liquid Biopsy of Cancer: A Multimodal Diagnostic Tool in Clinical Oncology. Ther. Adv. Med. Oncol. 2018, 10, 1758835918794630. [CrossRef]

10. Raimondi, L.; de Luca, A.; Gallo, A.; Costa, V.; Russelli, G.; Cuscino, N.; Manno, M.; Raccosta, S.; Carina, V.; Bellavia, D.; et al. Osteosarcoma Cell-Derived Exosomes Affect Tumor Microenvironment by Specific Packaging of Micrornas. Carcinogenesis 2019. [CrossRef]

11. Pantel, K.; Alix-Panabieres, C. Real-Time Liquid Biopsy in Cancer Patients: Fact or Fiction? Cancer Res. 2013, 73, 6384-6388. [CrossRef] [PubMed]

12. Raimondi, L.; de Luca, A.; Costa, V.; Amodio, N.; Carina, V.; Bellavia, D.; Tassone, P.; Pagani, S.; Fini, M.; Alessandro, R.; et al. Circulating Biomarkers in Osteosarcoma: New Translational Tools for Diagnosis and Treatment. Oncotarget 2017, 8, 100831-100851. [CrossRef] [PubMed]

13. Li, X.; Seebacher, N.A.; Hornicek, F.J.; Xiao, T.; Duan, Z. Application of Liquid Biopsy in Bone and Soft Tissue Sarcomas: Present and Future. Cancer Lett. 2018, 439, 66-77. [CrossRef] [PubMed]

14. Li, Y.S.; Liu, Q.; Tian, J.; He, H.B.; Luo, W. Angiogenesis Process in Osteosarcoma: An Updated Perspective of Pathophysiology and Therapeutics. Am. J. Med. Sci. 2019, 357, 280-288. [CrossRef] [PubMed]

15. Mohseny, A.B.; Cai, Y.; Kuijjer, M.; Xiao, W.; van den Akker, B.; de Andrea, C.E.; Jacobs, R.; Dijke, P.T.; Hogendoorn, P.C.; Cleton-Jansen, A.M. The Activities of Smad and Gli Mediated Signalling Pathways in High-Grade Conventional Osteosarcoma. Eur. J. Cancer 2012, 48, 3429-3438. [CrossRef]

16. Thery, C.; Amigorena, S.; Raposo, G.; Clayton, A. Isolation and Characterization of Exosomes from Cell Culture Supernatants and Biological Fluids. Curr. Protoc. Cell Biol. 2006, 30, 3-22. [CrossRef]

17. Bolger, A.M.; Lohse, M.; Usadel, B. Trimmomatic: A Flexible Trimmer for Illumina Sequence Data. Bioinformatics 2014, 30, 2114-2120. [CrossRef]

18. Langmead, B. Aligning Short Sequencing Reads with Bowtie. Curr. Protoc. Bioinform. 2010, 32, 11-17. [CrossRef]

19. Friedlander, M.R.; Mackowiak, S.D.; Li, N.; Chen, W.; Rajewsky, N. Mirdeep2 Accurately Identifies Known and Hundreds of Novel Microrna Genes in Seven Animal Clades. Nucleic Acids Res. 2012, 40, 37-52. [CrossRef]

20. Coulter, S.J. Mitigation of the Effect of Variability in Digital Pcr Assays through Use of Duplexed Reference Assays for Normalization. Biotechniques 2018, 65, 86-91. [CrossRef]

21. Agarwal, V.; Bell, G.W.; Nam, J.W.; Bartel, D.P. Predicting Effective Microrna Target Sites in Mammalian Mrnas. Elife 2015, 4, e05005. [CrossRef] [PubMed]

22. Chen, E.Y.; Tan, C.M.; Kou, Y.; Duan, Q.; Wang, Z.; Meirelles, G.V.; Clark, N.R.; Ma'ayan, A. Enrichr: Interactive and Collaborative Html5 Gene List Enrichment Analysis Tool. BMC Bioinform. 2013, 14, 128. [CrossRef] [PubMed]

(C) 2019 by the authors. Licensee MDPI, Basel, Switzerland. This article is an open access article distributed under the terms and conditions of the Creative Commons Attribution (CC BY) license (http://creativecommons.org/licenses/by/4.0/). 